\section{Lipids and brain: PUFA metabolism, function and protection against diseases}

The 2007 session of the "Journées Chevreul", a scientific event annually organised by the AFECG (Association Française pour I'Etude des Corps Gras) has been held during 2 days in Paris as an AFECG-DGF (Deutsche Gesellschaft für Fettwissenschaft) joint meeting under the umbrella of EuroFedLipid. We have been pleased to welcome 150 delegates, including more than $20 \%$ of PhD-students, representing 13 countries.

Scientifically devoted to "Lipids and Brain", this successful meeting offered a high quality program including 20 speakers and a few posters. The latest basis finding on the role of polyunsaturated fatty acids (PUFA) in the brain, essentially omega-3 (n-3) were exposed and discussed, with two major questions:

- What positive roles can be presently attributed to n-3 PUFA in early life development and aging, on pathophysiology and prevention of brain diseases?

- What do we know from epidemiological studies on the role of lipid nutrition and PUFA intake on brain functions and human behaviour?

The final part of the meeting focused on the scientific incoming efforts to provide n-3 LC-PUFA to human beings.

An interesting issue of this meeting has been that studies of omega-3 impact on the brain were not limited to the Long chains ( $n$-3 LC-PUFA), some of them reported also the growing evidence of interest of the precursor: Alpha-Linolenic Acid (ALA), provided by dietary vegetable oils (rapeseed and nuts) on protection in early life or against brain diseases. Interestingly, there are now epidemiological studies making the proof of links between dietary omega-3 (LC and precursor) status and the development of mood disorders, brain pathology or Alzheimer diseases.

Internationally recognized Scientists in the field of essential fatty acids have focused on new discoveries, burning questions and latest knowledge. One of the highlights of these days has been the "Medaille Chevreul" lecture given by a leader in this scientific field: Dr S. Rapoport, Bethesda, USA, who emphasized the recent developments of PUFA metabolism in brain and liver and its interplay and regulation by the diet. Just a few words from Dr Rapoport conclusions (see paper): "...In unanesthetized adult rats fed an n-3 PUFA "adequate" diet containing $4.6 \%$ (of total fatty acids) Alpha Linolenic Acid as its only n-3 PUFA, the liver secretes DHA derived from circulating ALA ten-times faster than the brain consumes DHA; thus the liver is capable of supplying all the brain's DHA..."

Most of the presentations are reported in the present dossier plus the poster's abstracts. The conclusions of the Vice-President of the AFECG provided a step by step summary of the results presented along the sessions.

Health professionals, nutritionists, food scientists from academy and public institutions as well as food industrialists active on research, development and marketing took part to this conference. The venue provided excellent networking facilities for delegates coming from different spheres of activity related to lipid nutrition and health aspects.

We are very grateful to speakers, participants, organisers and sponsors who made this symposium a great success.

The organisers gratefully acknowledge support by the following companies and institutions: Saint-Hubert/Marie, Lesieur, ONIDOL, PRONOVA/EPAX, CRODA, Van-deputte, Greentech/ agroindustries-Amazonica, Alliance, Pileje, LERO.

Bernadette Delplanque, CR Inserm AFECG President
DOSSIER 